\title{
Canadian oncogenic human papillomavirus cervical infection prevalence: Systematic review and meta-analysis
}

\author{
Andrea C Tricco ${ }^{1 *}$, Carmen H Ng${ }^{2}$, Vladimir Gilca ${ }^{3}$, Andrea Anonychuk ${ }^{4}$, Ba' Pham $^{5,6}$ and Shirra Berliner ${ }^{7}$
}

\begin{abstract}
Background: Oncogenic human papillomavirus (HPV) infection prevalence is required to determine optimal vaccination strategies. We systematically reviewed the prevalence of oncogenic cervical HPV infection among Canadian females prior to immunization.
\end{abstract}

Methods: We included studies reporting DNA-confirmed oncogenic HPV prevalence estimates among Canadian females identified through searching electronic databases (e.g., MEDLINE) and public health websites. Two independent reviewers screened literature results, abstracted data and appraised study quality. Prevalence estimates were meta-analyzed among routine screening populations, HPV-positive, and by cytology/histology results.

Results: Thirty studies plus 21 companion reports were included after screening 837 citations and 120 full-text articles. Many of the studies did not address non-response bias (74\%) or use a representative sampling strategy (53\%).

Age-specific prevalence was highest among females aged $<20$ years and slowly declined with increasing age. Across all populations, the highest prevalence estimates from the meta-analyses were observed for HPV types 16 (routine screening populations, 8 studies: 8.6\% [95\% confidence interval 6.5-10.7\%]; HPV-infected, 9 studies: 43.5\% [28.7-58.2\%]; confirmed cervical cancer, 3 studies: 48.8\% [34.0-63.6\%]) and 18 (routine screening populations, 8 studies: 3.3\% [1.5-5.1\%]; HPV-infected, 9 studies: 13.6\% [6.1-21.1\%], confirmed cervical cancer, 4 studies: 17.1\% [6.427.9\%].

Conclusion: Our results support vaccinating females $<20$ years of age, along with targeted vaccination of some groups (e.g., under-screened populations). The highest prevalence occurred among HPV types 16 and 18, contributing a combined cervical cancer prevalence of $65.9 \%$. Further cancer protection is expected from crossprotection of non-vaccine HPV types. Poor study quality and heterogeneity suggests that high-quality studies are needed.

Keywords: human papillomavirus, systematic review, meta-analysis, Bethesda system, cervical cancer

\section{Background}

Human Papillomavirus (HPV) is one of the most prevalent sexually transmitted infections in the world [1]. Over 100 virus genotypes have been identified and at least 13 are classified as oncogenic or "high-risk" (HR) because they are known to cause cervical cancer or other genital cancers $[2,3]$. The association between HR

\footnotetext{
* Correspondence: triccoa@smh.ca

'Li Ka Shing Knowledge Institute, St Michael's Hospital, (38 Shuter Street), Toronto, Ontario, (M5B 1T8), Canada

Full list of author information is available at the end of the article
}

HPV infection and cervical cancer has been well established in the literature with HR HPV DNA detected in nearly $100 \%$ of all cervical cancers [4-6].

For nearly half a century, cervical cancer prevention programs consisted mainly of cervical cancer screening for early detection of pre-cancerous lesions [7]. Although screening programs are effective, [8] cervical cancer remains a problem due to a large portion of women who remain unscreened or under-screened, [9-14] as well as false negative results on the Papanicolau test $[15,16]$. In 2009, an estimated 1,300 Canadian

\section{Biomed Central}


women developed cancer of the cervix and 390 died as a result of this disease [8]. The recent introduction of two prophylactic vaccines $[17,18]$ offer further reduction of $\mathrm{HPV}$, yet the most optimal primary and secondary prevention strategies remain unclear $[19,20]$.

Baseline prevalence data is necessary to inform optimal prevention programs and evaluate current and future prevention strategies [21,22]. Since HPV is not a reportable disease in Canada, data on HPV prevalence are widely scattered in the literature and mainly reported among specific populations. We aimed to consolidate the prevalence of DNA-confirmed cervical HPV infection among Canadian females prior to immunization programs through a systematic review and metaanalysis.

\section{Methods}

A systematic review protocol was compiled based on guidelines from The Cochrane Collaboration [23] and the Preferred Reporting Items for Systematic Reviews and Meta-Analyses [24]. The protocol is available upon request.

\section{Eligibility criteria and study selection}

Studies reporting DNA-confirmed (i.e., through hybrid capture I or hybrid capture II (HCI or HCII) or polymerase chain reaction (PCR)) HPV prevalence estimates among Canadian females who were not previously vaccinated against HPV were included. Authors of studies not reporting the sample size were contacted and the study was excluded if the sample size could not be obtained. Inclusion was not limited by study design, publication status, year of dissemination or language of dissemination.

In order to ensure reliability, a training exercise was conducted prior to commencing the screening process. Two independent reviewers screened the search results for inclusion using a pre-defined relevance criteria form, obtained the full-text of potentially relevant articles and screened them to determine inclusion. Discrepancies were resolved by discussion or the involvement of a third reviewer.

\section{Information sources and search}

Medical Subject Headings and text words related to the prevalence of HPV cervical infection among Canadian females were used to search MEDLINE (OVID interface, 1950 to October week 5, 2009), EMBASE (OVID interface, 1980 to 2009 week 44), The Cochrane Library (Wiley interface), and POPLINE (Knowledge for health interface 1970 to Nov 2, 2009). The electronic database search was supplemented by conducting a targeted search of Canadian public health websites (e.g., Public Health Agency of Canada, Health
Canada, Institut National de Sante Publique du Quebec), websites of organizations that produce guidelines (e.g., Canadian Agency for Technologies in Health, Society of Obstetrics and Gynecologists of Canada), and vaccine manufacturer websites (e.g., GlaxoSmithKline, Merck). Furthermore, general Internet searches were conducted in Google and the first 60 unique hits were scanned. In addition, the reference lists of included studies were scanned, the authors' personal files were searched, and HPV experts in public health and industry were emailed to ensure that all potentially relevant data was obtained.

An experienced information specialist conducted all of the literature searches. The search strategy for the main (MEDLINE) search is presented in Additional File 1. It was peer reviewed by another information scientist using Peer Review of Electronic Search Strategies (PRESS) [25]. The main search was modified for the other databases, as necessary (full search strategies for the other databases available upon request).

\section{Data collection process and data items}

A draft data abstraction form was developed, piloted, and modified as necessary. Two reviewers abstracted all of the data using the standardized data abstraction form, independently. Discrepancies were resolved by discussion or the involvement of a third reviewer.

The data abstracted included study characteristics, (e. g., study design, period of data collection, sample size, setting, province or territory and city of study conduct), participant characteristics (e.g., population, mean age and standard deviation), HPV detection method (e.g., HC-I or HC-II, PCR), PCR primers (e.g., MY09, MY11), number of HPV positive, as well as the overall HPV prevalence. As done in previous reviews of HPV prevalence, [26-28] we focused on HR HPV types commonly recognized to be associated with cervical cancer. These included the following HPV types: 16, 18, 31, 33, 35, 39, $45,51,52,56,58,59$, and 68 [3]. Specimens that were excluded from study analysis due to inadequate specimen integrity (e.g., negative for $\beta$-globin) were not included in the sample size.

Four of the included studies directly compared prevalence results for different tests (e.g., PCR versus HC-II) or methods (e.g., wood versus plastic spatula; cotton swab versus cytobrush) [29-32]. For these studies, prevalence results for the test or method found to be the most sensitive were abstracted. These included HC-II, [31,32] plastic spatula, [29] and cytobrush [30]. In some instances, multiple study publications reported data from the same population (i.e., companion reports). When this occurred, the report with the largest sample size was included and the other report(s) was used for supplementary data. 
A commonly used and validated quality tool for observational studies does not exist [33]. As such, a generic methodological quality tool was developed and applied to all of the included studies, regardless of study design using a similar format that was used in a previous systematic review [34]. One item was added from The Cochrane Collaboration's Risk of Bias Tool on outcome reporting bias, which ensures that all outcomes that were assessed are reported in the study report [23]. The final tool consisted of the sampling strategy, sensitivity of sampling, timing of sampling, non-response bias, outcome reporting bias, and conflict of interest (Additional File 2).

\section{Age-specific prevalence synthesis}

Studies reporting the age-specific prevalence of HR HPV infection were plotted and variation due to timing of data collection, HPV detection methods, and study location was noted. In order to ensure consistency across studies, only studies that collected samples in routine screening (i.e., routine Papanicolau test) were included in the plot. These results were described narratively and not combined via meta-analysis to view the wide heterogeneity observed across studies.

\section{Prevalence meta-analyses}

HPV prevalence among routine screening populations was defined as the proportion of individuals who were positive for HR HPV infection divided by the total population tested for HPV infection. HPV type-specific prevalence among routine screening populations was defined as the proportion of females testing positive for the specific HR HPV type among all of those testing for HPV infection. These analyses only included studies of females engaged in routine screening.

HR HPV prevalence and HR HPV type-specific prevalence was also calculated for those testing positive for HPV infection. These analyses included studies of females engaged in routine screening, as well as those returning after a previous abnormal cytological test [35].

HPV type-specific prevalence by cytology or histology was defined as the proportion of individuals testing positive for the specific HR HPV type broken down by the cytological and/or histological category according to the 2001 Bethesda System (Additional File 3) [36]. Studies using routine screening, those returning after a previous abnormal cytological test, as well as those receiving a biopsy were included in this analysis. Individuals diagnosed with squamous cell carcinoma of the cervix and/or aden/adenosquamous carcinoma of the cervix were included in the confirmed cancer category.

Pooled estimates of type-specific prevalence of HR HPV infection were derived using a random-effects model [37]. The 95\% CIs were derived based on a normal distribution. In order to produce conservative prevalence estimates and corresponding 95\% CIs, studies reporting zero for the number of positives were imputed as being 0.5. Each HR HPV type was considered individually; hence prevalence estimates might include concomitant infection with other HPV types. All analyses were conducted in SAS 9.1 software (SAS Institute Inc., Cary, NC, USA).

\section{Results}

\section{Literature search}

The literature search resulted in a total of 837 citations (i.e., titles and abstracts). Thirty study reports [29-32,35,38-62] plus 21 companion reports [27,28,63-81] fulfilled the inclusion criteria and were included after screening 120 full-text articles (Figure 1).

\section{Study characteristics}

Data were collected from 1960 [42] to 2007 [48] (Table 1). Sample sizes varied from 46 [38] to 9,620 females [54]. Most of the included studies reported the actual number of HPV positive, except for one study, which reported weighted estimates [39]. Twenty-eight of the included studies were published in journals and two were theses $[48,55]$. Six studies reported infection with multiple HPV types (i.e., multiple infection), which was an average of $17.1 \%$ (median $4.4 \%$ ) and ranged from $1.2 \%$ to $62.0 \%[31,39,40,49,58,62]$.

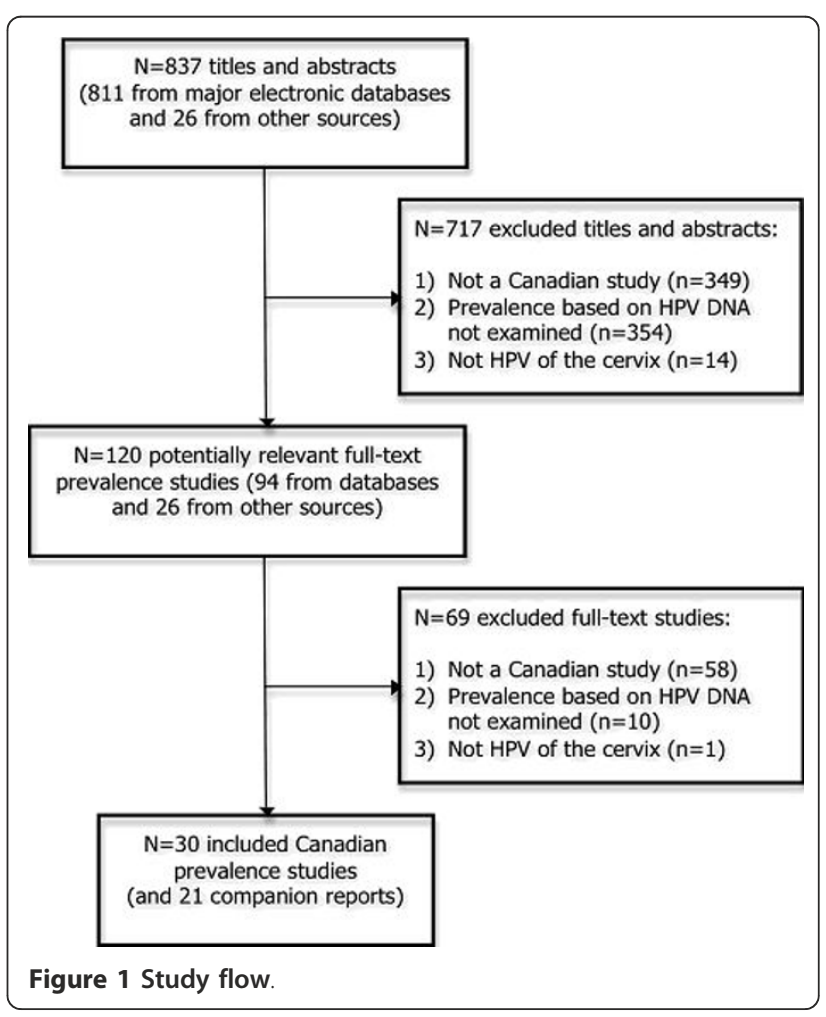


Overall, 74\% (17/23) did not address non-response bias, $53 \%(16 / 30)$ did not use a representative sampling strategy (i.e., they included individuals with risk factors for HPV and their results cannot be generalized to the general population), 17\% (5/30) did not use adequate timing of sampling (e.g., retrospective instead of prospective), and $17 \%$ (5/30) did not use sensitive sampling techniques (e.g. hybridization). Seven studies out of 30 were not relevant to the non-response bias and response rate methodological quality items because they included data from cancer biopsies (Additional File 4) [38-40,43,45,46,51]. Excluding these studies, some of the others $(35 \%, 8 / 23)$ did not report the response rate or used an inappropriate approach to calculate the response rate $(9 \%, 2 / 23)$. Individual methodological quality components for each study are reported in Table 1.

\section{Age-specific HPV prevalence}

The age-specific prevalence was generally highest among females less than 20 years of age and slowly declined as age increased, despite widespread heterogeneity across studies (Figure 2). HPV prevalence among females less than 20 years of age [31,48,50,58] ranged from $14.1 \%$ among university students [58] to $46.9 \%$ among Inuit females from Nunavut [48]. For women aged 20-25 years, HPV prevalence ranged from $13.0 \%$ among university students [58] to $24.0 \%$ among the general population of Ontario [31]. The prevalence among Nunavut women aged $21-30$ years was $31.3 \%$ [50] and the prevalence of Inuit women from Quebec aged 20-29 years was $24.5 \%$ [48]. Ontario women aged 25-29 years had a prevalence of $16.4 \%$ [31] and Newfoundland women aged 25-34 years had a prevalence of $11.8 \%$ [57].

HPV prevalence among women aged 30-39 years [31,48,50,54,57] ranged from $7.0 \%$ in Newfoundland [54] to $17.2 \%$ among Inuit women from Quebec [48]. For women aged 40-49 years, $[31,48,54]$ the prevalence ranged from $4.0 \%$ in Newfoundland [54] to $8.3 \%$ in Ontario [31]. Prevalence among women aged 50-59 years $[32,48,54]$ ranged from $2.5 \%$ in Newfoundland [54] to $8.9 \%$ among women aged 50-54 years in Ontario [32]. For women aged 60-69 years, $[48,54]$ the prevalence ranged from $1.2 \%$ in Newfoundland [54] to $12.5 \%$ among Inuit women from Quebec [48]. Finally, the prevalence among Ontario women aged greater than 60 was $11.3 \%$ [32]. Overall, age-specific HPV prevalence estimates were higher for other populations (i.e., those testing positive for HPV and HPV prevalence by histology/cytology) compared to the routinely screened populations (data not shown).

\section{Meta-analysis results}

The meta-analysis results of HPV prevalence among routine screening populations are presented in Figure 3 and the three HPV types with the highest prevalence (HPV$16,-18$, and -52) are highlighted here. The studies included in these analyses were conducted between 1990 and 2007, included an average of 1,019 individuals, and varied in their study location (British Columbia (BC), Manitoba (MB), ON, QC) [31,39,48,49,55,58-60,62]. For HPV-16, the pooled prevalence was $8.6 \%$ (95\% CI: $6.5-$ $10.7 \%)$; the lowest prevalence estimate was reported among university students (4.8\%); [58] while the highest prevalence estimate was among participants from a lowincome, inner city population (12.6\%) [62]. The pooled prevalence for HPV-18 was 3.3\% (95\% CI: $1.5-5.1 \%$ ) $[31,39,48,49,55,58-60,62]$. The lowest prevalence estimate was $0 \%$ among university students, [60] while the highest prevalence was $11.8 \%$ among a low-income, inner city population [62]. Four studies reported HPV prevalence for HPV-52 and their pooled prevalence was $2.8 \%$ (95\% CI: $1.2-4.4 \%)[39,48,49,58]$. The lowest prevalence estimate was $0 \%$ among a sample of university students, [58] while the highest prevalence estimate was $10.8 \%$ among women presenting to an sexually transmitted disease clinic [49].

The meta-analysis results for HPV prevalence among HPV-positive are presented in Figure 4 and the three $\mathrm{HPV}$ types with the highest prevalence (HPV-16, -18 , and -31) are highlighted here. The studies included in these analyses were conducted between 1990 and 2007, included an average of 985 individuals, and varied in their study location (e.g., BC, MB, ON, QC) $[31,35,39,48,55,58-60,62]$. For HPV-16, the pooled prevalence from these nine studies was $43.5 \%$ (95\% CI: 28.7-58.2\%). The lowest prevalence was among Inuit women (19.4\%) [48] and the highest prevalence was among participants with an abnormal Pap test $(76.0 \%)$ [35]. For HPV-18, the pooled prevalence from these nine studies was $13.6 \%$ (95\% CI: 6.1-21.1\%). The lowest prevalence was among university students (0\%), [60] while the highest prevalence was from a general population sample from a low-income, inner-city area (36.3\%) [62]. For HPV-31, the pooled prevalence was $10.1 \%$ (95\% CI: 4.8-15.3\%), [35,39,49,58-60,62] and ranged from $2.3 \%$ among the general population [39] to $24.8 \%$ among participants with an abnormal Pap test [35].

The meta-analysis results according to the Bethesda classification system are presented in Table 2. The prevalence generally increased as the type of cervical lesion approached cervical cancer. The observed prevalence of combined HR HPV types for those with negative cytology or histology was $40.5 \%$ (95\% CI: $26.4 \%-54.6 \%$ ); 15.7\% (95\% CI: 9.3\%-22.0\%) for benign lesions; $40.5 \%$ (95\% CI: $26.4 \%-54.6 \%$ ) for ASC-US; 73.6\% (95\% CI: $55.3 \%-91.8 \%$ ) for LSIL; and 89.9\% (95\% CI: 84.8\%-95.1\%) for HSIL. 
Table 1 Study characteristics

\begin{tabular}{|c|c|c|c|c|c|c|c|c|c|}
\hline Reference & $\begin{array}{l}\text { Study population; year } \\
\text { of data collection; } \\
\text { province(s) }\end{array}$ & $\begin{array}{l}\text { Sample } \\
\text { size }\end{array}$ & $\begin{array}{l}\text { Mean age } \\
\text { (SD) or } \\
\text { age range }\end{array}$ & $\begin{array}{l}\text { Method of } \\
\text { detection }\end{array}$ & $\begin{array}{c}\# \\
\text { cases‡ }\end{array}$ & $\begin{array}{c}\text { HPV } \\
\text { types } \\
\text { detected }\end{array}$ & $\begin{array}{c}\text { Overall } \\
\text { HPV } \\
\text { prevalence } \\
\text { (any type)† }\end{array}$ & $\begin{array}{c}\mathrm{HR} \text { HPV } \\
\text { prevalence }\end{array}$ & $\begin{array}{l}\text { Methodological } \\
\text { quality } \\
\text { components }\end{array}$ \\
\hline $\begin{array}{c}\text { Peng } \\
1988 \text { [30] }\end{array}$ & $\begin{array}{c}\text { Patients with abnormal } \\
\text { pap smears } \\
\text { Dates NR } \\
\text { Ontario }\end{array}$ & 200 & NR & $\begin{array}{c}\text { In-situ } \\
\text { hybridization }\end{array}$ & $\begin{array}{l}28 \\
(\mathrm{HR} 16, \\
18)\end{array}$ & $\begin{array}{l}\text { Specific } \\
\text { HPV } \\
\text { types } \\
\text { probed§ }\end{array}$ & NA & $\begin{array}{c}14 \% \\
(H R 16,18)\end{array}$ & $\begin{array}{c}\text { 1.N, 2.Y, 3.U, 4.N, } \\
5 . Y, 6 . N, 7 . Y\end{array}$ \\
\hline $\begin{array}{c}\text { Caussy } \\
1990[42]\end{array}$ & $\begin{array}{c}\text { Cases with cervical cancer, } \\
\text { controls with CIN without } \\
\text { cervical cancer } \\
\text { 1960-1986 } \\
\text { British Columbia }\end{array}$ & 141 & $35.6(24)$ & $\begin{array}{c}\text { In-situ } \\
\text { hybridization }\end{array}$ & 31 & HR-16, 18 & $22 \%$ & $17.0 \%$ & $\begin{array}{c}\text { 1.N, 2.Y, 3.U, 4.Y, } \\
5 . \mathrm{N}, 6 . \mathrm{N}, 7 . \mathrm{Y}\end{array}$ \\
\hline $\begin{array}{l}\text { Duggan } \\
1990 \text { [29] }\end{array}$ & $\begin{array}{c}\text { Patients referred to } \\
\text { colposcopy clinic } \\
\text { Dates NR } \\
\text { Alberta }\end{array}$ & 119 & NR & $\begin{array}{c}\text { Dot blot } \\
\text { hybridization }\end{array}$ & $\begin{array}{c}28 \\
(\mathrm{HPV} \\
16 / 18 / \\
33)\end{array}$ & $\begin{array}{l}\text { HR-16, } \\
18,33 \\
\text { LR-6, } 11\end{array}$ & NA & $\begin{array}{l}23.5 \% \\
(\mathrm{HPV} 16 / \\
18 / 33)\end{array}$ & $\begin{array}{c}\text { 1.N, 2.Y, 3.U, 4.N, } \\
\text { 5.Y, 6.N, 7.U }\end{array}$ \\
\hline $\begin{array}{l}\text { Rohan } \\
1991 \text { [60] }\end{array}$ & $\begin{array}{c}\text { University Students: } \\
\text { Routine Screening } \\
1990 \\
\text { Ontario }\end{array}$ & 105 & $\begin{array}{l}23 \text { (median } \\
\text { age) }\end{array}$ & PCR (E7/E6) & 19 & $\begin{array}{l}\text { HR-16, } \\
18,33 \\
\text { LR-6, } 11\end{array}$ & $12.3 \%$ & $10.5 \%$ & $\begin{array}{c}1 . Y, 2 . Y, 3 . Y, 4 . N, \\
5 . Y, 6 . Y, 7 . Y\end{array}$ \\
\hline $\begin{array}{c}\text { Guijon } \\
1993 \text { [47] }\end{array}$ & $\begin{array}{c}\text { Patients referred to } \\
\text { colposcopy clinic } \\
\text { 1985-1990 } \\
\text { Manitoba }\end{array}$ & 409 & 25.7 (NR) & $\begin{array}{c}\text { In-situ } \\
\text { hybridization }\end{array}$ & 280 & $\begin{array}{c}\text { HR-16, } \\
18,33 \\
\text { LR-6, } 11\end{array}$ & NA & $\begin{array}{c}68.5 \% \\
\text { (HPV 16/18) }\end{array}$ & $\begin{array}{c}\text { 1.N, 2.Y, 3.Y, 4.N, } \\
5 . Y, 6 . N, 7 . Y\end{array}$ \\
\hline $\begin{array}{c}\text { Brisson } \\
1994 \text { [41] }\end{array}$ & $\begin{array}{c}\text { Patients referred to } \\
\text { colposcopy clinic } \\
\text { 1988-1989 } \\
\text { Quebec }\end{array}$ & 1140 & & Southern Blot & $\begin{array}{l}28.2 \\
(6.6)\end{array}$ & $\begin{array}{l}\text { HR-16, } 18 \\
\text { LR-6, } 11\end{array}$ & NA & $\begin{array}{c}24.3 \% \\
(\mathrm{HPV}-16)\end{array}$ & $\begin{array}{c}\text { 1.N, 2.Y, 3.Y, 4.N, } \\
5 . Y, 6 . N, 7 . Y\end{array}$ \\
\hline $\begin{array}{c}\text { Bosch } \\
1995[38]\end{array}$ & $\begin{array}{c}\text { Tumor biopsies of invasive } \\
\text { cervical cancer } \\
1989-1992 \\
\text { Province NR }\end{array}$ & 46 & NR & $\begin{array}{l}\text { PCR (MY09/ } \\
\text { MY11) }\end{array}$ & 43 & HR-16 & $93.5 \%$ & $91.3 \%$ & $\begin{array}{c}\text { 1.NA, 2.Y, 3.NA, } 4 . \\
N, 5 . N, 6 . Y, 7 . Y\end{array}$ \\
\hline $\begin{array}{l}\text { Duggan } \\
1995[43]^{*} \\
{[27,68]}\end{array}$ & $\begin{array}{c}\text { Cervical cancer tumour } \\
\text { biopsies } \\
\text { 1970-1990 } \\
\text { Alberta }\end{array}$ & 76 & $47.3(16.7)$ & $\begin{array}{l}\text { Dot blot } \\
\text { supplemented } \\
\text { by PCR }\end{array}$ & 53 & All & $70.0 \%$ & $65.8 \%$ & $\begin{array}{l}\text { 1.NA, 2.Y, 3.NA } 4 . \\
Y, 5 . N, 6 . Y, 7 . Y\end{array}$ \\
\hline $\begin{array}{c}\text { Franco } \\
1996[46]\end{array}$ & $\begin{array}{c}\text { Cervical cancer tumour } \\
\text { biopsies } \\
\text { 1983-1990 } \\
\text { Quebec } \\
\end{array}$ & 69 & NR & $\begin{array}{l}\text { PCR (GP5 } \\
+/ G P 6+)\end{array}$ & 59 & $\begin{array}{l}\text { HR-16, } \\
18,31 \\
33,35 \\
\text { LR-6, } 11 \\
\end{array}$ & $85.5 \%$ & $\begin{array}{c}40.6 \% \\
\text { (HPV 16/18) }\end{array}$ & $\begin{array}{c}\text { 1.NA, 2.Y, 3.NA, } 4 . \\
\text { N, 5.N, 6.Y, 7.Y }\end{array}$ \\
\hline $\begin{array}{c}\text { Young } \\
1997 \text { [62] }\end{array}$ & $\begin{array}{c}\text { General Public (Low- } \\
\text { income, inner-city area): } \\
\text { Routine Screening } \\
\text { 1992-1995 } \\
\text { Manitoba }\end{array}$ & 1263 & $\begin{array}{l}73 \% \text { less } \\
\text { than } 30 \\
\text { years }\end{array}$ & $\begin{array}{l}\text { PCR (MY09/ } \\
\text { MY11) }\end{array}$ & 411 & $\begin{array}{l}\text { HR-16, } \\
18,31 \\
\text { LR-6, } 11\end{array}$ & $32.5 \%$ & $\begin{array}{c}18.0 \% \\
\text { (HPV 16/18) }\end{array}$ & $\begin{array}{c}\text { 1.N, 2.Y, 3.U, 4.N, } \\
\text { 5.Y, 6.Y, 7.Y }\end{array}$ \\
\hline $\begin{array}{l}\text { Duggan } \\
1997[44]^{*} \\
{[67,69,70]}\end{array}$ & $\begin{array}{c}\text { Cervical biopsies } \\
\text { NR } \\
\text { Alberta }\end{array}$ & 525 & 28.0 & PCR & 249 & $\begin{array}{l}\text { HR-16, } \\
18,31 \\
33,35 \\
\text { LR-6, } 11 \\
\end{array}$ & $47.4 \%$ & $\begin{array}{c}28.0 \% \\
\text { (HPV 16/18) }\end{array}$ & $\begin{array}{c}\text { 1.N, 2.Y, 3.U, 4.Y, } \\
5 . Y, 6 . Y, 7 . \cup\end{array}$ \\
\hline $\begin{array}{c}\text { Michael } \\
1999 \text { [55] }\end{array}$ & $\begin{array}{c}\text { University Students: } \\
\text { Routine Screening } \\
\text { NR } \\
\text { Ontario }\end{array}$ & 99 & NR & $\begin{array}{l}\text { PCR (MY09/ } \\
\text { MY11) }\end{array}$ & 40 & $\begin{array}{l}\text { HR-16, } \\
18,31 \\
33,35 \\
\text { LR-6, } 11\end{array}$ & $40.4 \%$ & $\begin{array}{c}10.1 \% \\
\text { (HPV 16/18) }\end{array}$ & $\begin{array}{c}1 . N, \text { 2.Y, 3.Y, 4.Y, } \\
5 . Y, 6 . Y, 7 . Y\end{array}$ \\
\hline $\begin{array}{c}\text { Hankins } \\
1999[49]^{*} \\
{[63,64,66,71]}\end{array}$ & $\begin{array}{c}\text { HIV positive } \\
\text { 1993-2000 } \\
\text { Multiple provinces }\end{array}$ & 375 & 32.5 & $\begin{array}{l}\text { PCR (MY09/ } \\
\text { MY11) }\end{array}$ & 252 & HR-16, 18 & $67.2 \%$ & $49.1 \%$ & $\begin{array}{c}1 . N, 2 . Y, 3 . U, 4 . Y, \\
5 . Y, 6 . Y, 7 . Y\end{array}$ \\
\hline $\begin{array}{l}\text { Richardson } \\
2000 \text { [58] }\end{array}$ & $\begin{array}{c}\text { University Students: } \\
\text { Routine Screening } \\
1992-1993 \\
\text { Quebec }\end{array}$ & 375 & $18-24$ & $\begin{array}{l}\text { PCR (MY09/ } \\
\text { MY11) }\end{array}$ & 85 & $\begin{array}{l}\text { HR- }[11] \\
\text { LR-6,11,53 }\end{array}$ & $22.7 \%$ & $11.8 \%$ & $\begin{array}{c}1 . N, 2 . Y, 3 . Y, 4 . N, \\
5 . Y, 6 . Y, 7 . U\end{array}$ \\
\hline $\begin{array}{c}\text { Sellors } \\
\text { 2000a }[31]^{*} \\
{[65,74,79]}\end{array}$ & $\begin{array}{c}\text { General Public: Routine } \\
\text { Screening } \\
\text { 1998-1999 } \\
\text { Ontario }\end{array}$ & $\begin{array}{c}955(\mathrm{HC}- \\
\text { II); } \\
824 \\
\text { (PCR) }\end{array}$ & $15-49$ & $\begin{array}{l}\text { PCR (MY09/ } \\
\text { MY11; } \\
\text { HC-II }\end{array}$ & 110 & $\begin{array}{l}\text { HR- [11] } \\
\text { LR- [16] }\end{array}$ & $13.3 \%$ & $\begin{array}{l}12.7 \% \\
(\mathrm{HC}-\mathrm{II})\end{array}$ & $\begin{array}{c}1 . Y, 2 . Y, 3 . Y, 4 . Y, \\
5 . Y, 6 . Y, 7 . Y\end{array}$ \\
\hline
\end{tabular}


Table 1 Study characteristics (Continued)

\begin{tabular}{|c|c|c|c|c|c|c|c|c|c|}
\hline $\begin{array}{c}\text { Sellors } \\
\text { 2000b [35] }\end{array}$ & $\begin{array}{c}\text { Patients recalled after an } \\
\text { Abnormal Pap on Routine } \\
\text { Screening } \\
\text { 1996-1997 } \\
\text { Ontario }\end{array}$ & 200 & $31.5(9.4)$ & HC-II/PCR (L1) & 125 & $\begin{array}{l}\text { HR- }[13] \\
\text { LR- } \\
6,11,42,53\end{array}$ & $62.5 \%$ & $90.3 \%$ HSIL & $\begin{array}{c}1 . N, 2 . Y, 3 . Y, 4 . N \\
5 . Y, 6 . Y, 7 . Y\end{array}$ \\
\hline $\begin{array}{l}\text { Ratnam } \\
2000 \text { [57] }\end{array}$ & $\begin{array}{c}\text { General Public: Routine } \\
\text { Screening } \\
1996-1998 \\
\text { Newfoundland }\end{array}$ & 2098 & 30 (NR) & $\mathrm{HC}-\mathrm{I} / \mathrm{HC}-\mathrm{II}$ & $\begin{array}{c}227 \\
(H R 13)\end{array}$ & $\begin{array}{l}\text { HR- }[13] \\
\text { LR- } \\
6,11,42,53\end{array}$ & NA & $10.8 \%$ & $\begin{array}{c}1 . Y, 2 . Y, 3 . Y, 4 . Y \\
5 . Y, 6 . Y, 7 . Y\end{array}$ \\
\hline $\begin{array}{c}\text { Feoli- } \\
\text { Fonseca } \\
2001[45]^{*} \\
{[77]}\end{array}$ & $\begin{array}{c}\text { Biopsies suspected of HPV } \\
\text { Dates NR } \\
\text { Quebec }\end{array}$ & 691 & 26.1 & $\begin{array}{l}\text { PCR (MY09/ } \\
\text { MY11/GP5 } \\
\text { +/GP6+) }\end{array}$ & 484 & HR- [13] & $70.0 \%$ & $\begin{array}{c}92.0 \%(\mathrm{CIN} \\
\text { III) } \\
100 \% \\
\text { (carcinoma } \\
\text { in situ) }\end{array}$ & $\begin{array}{l}\text { 1.NA, 2.Y, 3.NA, } 4 . \\
Y, 5 . N, 6 . Y, 7 . Y\end{array}$ \\
\hline $\begin{array}{c}\text { Healey } \\
2001[50]^{*} \\
{[72,73]}\end{array}$ & $\begin{array}{c}\text { General Public (Inuit): } \\
\text { Routine Screening } \\
1999 \\
\text { Nunavut }\end{array}$ & 1290 & $13-79$ & $\mathrm{HC}-\|$ & 333 & HR- [13] & & $25.8 \%$ & $\begin{array}{c}\text { 1.Y, 2.Y, 3.Y, 4.Y, } \\
\text { 5.Y, 6.Y, 7.Y }\end{array}$ \\
\hline $\begin{array}{c}\text { Tran-Thanh } \\
2002[61]^{*} \\
{[80]}\end{array}$ & $\begin{array}{c}\text { Cases with SIL or invasive } \\
\text { cancer and controls with } \\
\text { normal cytology and no } \\
\text { history of cervical disease } \\
1998-2000 \\
\text { Quebec }\end{array}$ & 320 & $16-73$ & $\begin{array}{c}\text { PCR (MY09/ } \\
\text { MY11) }\end{array}$ & 206 & HR- [13] & $64.4 \%$ & & $\begin{array}{c}1 . N, 2 . Y, 3 . Y, 4 . N \\
5 . Y, 6 . Y, 7 . Y\end{array}$ \\
\hline $\begin{array}{c}\text { Sellors } 2002 \\
{[32]}\end{array}$ & $\begin{array}{c}\text { General Public: Routine } \\
\text { Screening } \\
1999-2000 \\
\text { Ontario }\end{array}$ & 156 & $\begin{array}{c}50 \text { and } \\
\text { over }\end{array}$ & $\mathrm{HCII} / \mathrm{PCR}$ & 13 & $\begin{array}{l}\text { HR- }[13] \\
\text { LR- }[14]\end{array}$ & NA & $\begin{array}{c}8.3 \% \\
(H C-I I)\end{array}$ & $\begin{array}{c}\text { 1.N, 2.Y, 3.N, 4.N, } \\
\text { 5.Y, 6.Y, 7.U }\end{array}$ \\
\hline $\begin{array}{c}\text { Richardson } \\
2003[59]^{*} \\
{[81]}\end{array}$ & $\begin{array}{c}\text { University Students: } \\
\text { Routine Screening } \\
\text { 1996-1998 } \\
\text { Quebec }\end{array}$ & 621 & $\begin{array}{l}83 \% \text { under } \\
\text { age } 27 \\
\text { years; } \\
\text { mean age } \\
23 \text { (NR) }\end{array}$ & $\begin{array}{c}\text { PCR (MY09/ } \\
\text { MY11) }\end{array}$ & 180 & HR- [13] & $29 \%$ & $21.8 \%$ & $\begin{array}{c}\text { 1.N, 2.Y, 3.U, 4.N, } \\
\text { 5.Y, 6.Y, 7.Y }\end{array}$ \\
\hline $\begin{array}{c}\text { Lytwyn } \\
2003[75]^{*} \\
{[53]}\end{array}$ & $\begin{array}{c}\text { Patients recalled after an } \\
\text { Abnormal Pap: ASCUS or } \\
\text { LSIL only } \\
\text { 1995-1998 } \\
\text { Ontario }\end{array}$ & 105 & $30.3(8.1)$ & $\mathrm{HC}-\|$ & $\begin{array}{c}57 \\
(H R 13)\end{array}$ & $\begin{array}{l}\text { HR- }[13] \\
\text { LR- }[14]\end{array}$ & NA & $54.3 \%$ & $\begin{array}{c}1 . Y, 2 . Y, 3 . Y, 4 . Y \\
\text { 5.Y, 6.Y, 7.Y }\end{array}$ \\
\hline $\begin{array}{c}\text { Koushik } \\
2005[52]^{*} \\
{[76,78]}\end{array}$ & $\begin{array}{l}\text { General Public: Cases with } \\
\text { CIN from colposcopy } \\
\text { clinics, controls with } \\
\text { normal pap from } \\
\text { screening. } \\
2001-2003 \\
\text { Quebec }\end{array}$ & $\begin{array}{l}357 \\
\text { cases, } \\
760 \\
\text { controls }\end{array}$ & $\begin{array}{l}32.0(9.1) \\
\text { Cases, } \\
31.7(10.0) \\
\text { Controls }\end{array}$ & $\begin{array}{c}\text { PCR (MY09/ } \\
\text { MY11) }\end{array}$ & 579 & HR- [13] & $51.8 \%$ & $41.8 \%$ & $\begin{array}{c}\text { 1.N, 2.Y, 3.Y, 4.Y, } \\
\text { 5.Y, 6.Y, 7.Y }\end{array}$ \\
\hline $\begin{array}{l}\text { Mayrand } \\
2006[54]\end{array}$ & $\begin{array}{c}\text { General Public: Routine } \\
\text { Screening } \\
\text { 2002-2004 } \\
\text { Quebec, Newfoundland }\end{array}$ & 9620 & $N R$ & $\mathrm{HCll}$ & 591 & $\begin{array}{l}\text { HR- }[13] \\
\text { LR- [24] }\end{array}$ & NA & $6.1 \%$ & $\begin{array}{c}1 . N, 2 . Y, 3 . Y, 4 . Y \\
5 . Y, 6 . Y, 7 . Y\end{array}$ \\
\hline $\begin{array}{l}\text { Ogilvie } \\
2007[56]\end{array}$ & $\begin{array}{c}\text { Sex trade workers or } \\
\text { women with a history of } \\
\text { alcohol or drug abuse } \\
2004-2005 \\
\text { British Columbia }\end{array}$ & 151 & 39.0 & $\mathrm{HC}-\mathrm{II}$ & $\begin{array}{c}43 \\
(\mathrm{HC} 13)\end{array}$ & HR- [13] & NA & $28.5 \%$ & $\begin{array}{c}\text { 1.N, 2.Y, 3.Y, 4.N, } \\
\text { 5.Y, 6.Y, 7.Y }\end{array}$ \\
\hline $\begin{array}{c}\text { Kapala } 2007 \\
{[51]}\end{array}$ & $\begin{array}{c}\text { General Public: Routine } \\
\text { Screening } \\
\text { Date NR } \\
\text { Ontario }\end{array}$ & 320 & $\begin{array}{l}73 \% \text { age } \\
30 \text { or } \\
\text { greater }\end{array}$ & $\mathrm{HC}-\mathrm{II}$ & $\begin{array}{c}92 \\
(H R 13)\end{array}$ & HR- [13] & NA & $28.8 \%$ & $\begin{array}{c}\text { 1.NA, 2.Y, 3.NA, } 4 . \\
N, 5 . Y, 6 . Y, 7 . U\end{array}$ \\
\hline $\begin{array}{c}\text { Antionishyn } \\
2008 \text { [40] }\end{array}$ & $\begin{array}{c}\text { Cervical biopsies } \\
\text { 1995-1998 } \\
\text { Saskatchewan }\end{array}$ & 1355 & NR & $\begin{array}{l}\text { PCR (GP5 } \\
+/ G P 6+)\end{array}$ & 753 & HR- [13] & $55.6 \%$ & $\begin{array}{c}56.8 \% \text { CIN } \\
\text { III (16 and } \\
18)\end{array}$ & $\begin{array}{c}\text { 1.NA, 2.Y, 3.NA, } 4 . \\
N, 5 . Y, 6 . Y, 7 . Y\end{array}$ \\
\hline $\begin{array}{l}\text { Hamlin- } \\
\text { Douglas } \\
2008[48]\end{array}$ & $\begin{array}{c}\text { General Public (Inuit): } \\
\text { Routine Screening } \\
\text { 2002-2007 } \\
\text { Quebec }\end{array}$ & 554 & $35.5(14.4)$ & $\begin{array}{c}\text { PCR (MY09/ } \\
\text { MY11) }\end{array}$ & 160 & All & $28.9 \%$ & $20.4 \%$ & $\begin{array}{c}1 . Y, 2 . Y, 3 . N, 4 . Y \\
\text { 5.Y, 6.Y, 7.Y }\end{array}$ \\
\hline
\end{tabular}


Table 1 Study characteristics (Continued)

\begin{tabular}{|c|c|c|c|c|c|c|c|c|c|}
\hline $\begin{array}{c}\text { Moore } \\
2009 \text { [39] }\end{array}$ & $\begin{array}{l}\text { Population-based } \\
\text { estimates weighted by } \\
\text { cytology } \\
2004 \\
\text { British Columbia }\end{array}$ & 4821 & $13-86$ & $\begin{array}{l}\text { PCR (GP5 } \\
+/ \text { GP6+) }\end{array}$ & 810 & All & $16.8 \%$ & $13.9 \%$ & $\begin{array}{l}\text { 1.NA, 2.Y, 3.NA, } 4 . \\
Y, 5 . Y, 6 . Y, 7 . N\end{array}$ \\
\hline
\end{tabular}

Notes: * major publication and other references are companion reports, $\neq$ number of women positive for human papillomavirus, $\dagger$ calculated by using the "number of cases" value as the numerator and the "sample size" as the denominator, expressed as a percent. Abbreviations: SD standard deviation, NR not reported, HC hybrid capture, PCR polymerase chain reaction.

The most prevalent HPV type among those classified as negative for intraepithelial lesion or malignancy was HPV-16 (7.9\%) and the prevalence for each of the other HR types was less than 5\%. Among those classified with LSIL, the three most prevalent types were HPV-16 (28.3\%), HPV-31 (7.3\%), and HPV-18 (5.8\%). Among those classified with HSIL, the three most prevalent types were HPV-16 (54.0\%), HPV-31 (14.2\%), and HPV-18 (7.9\%). Among those with confirmed cervical cancer, the highest prevalence was observed for HPV-16 (48.8\% [95\% CI: 34.0-63.6\%]) [38,43,61], followed by HPV-18 (17.1\% (95\% CI: 6.4-27.9\%) [38,42,43,61], and HPV-45 (7.7\%, [95\% CI: 2.4-13.0\%]) $[38,61]$.

\section{Sensitivity analysis}

We conducted a post hoc sensitivity analysis of the meta-analysis results for key study characteristics including study location, study design, HPV detection method, sample size, and methodological quality. Differences in prevalence estimates across the included studies were not identified (data not shown).

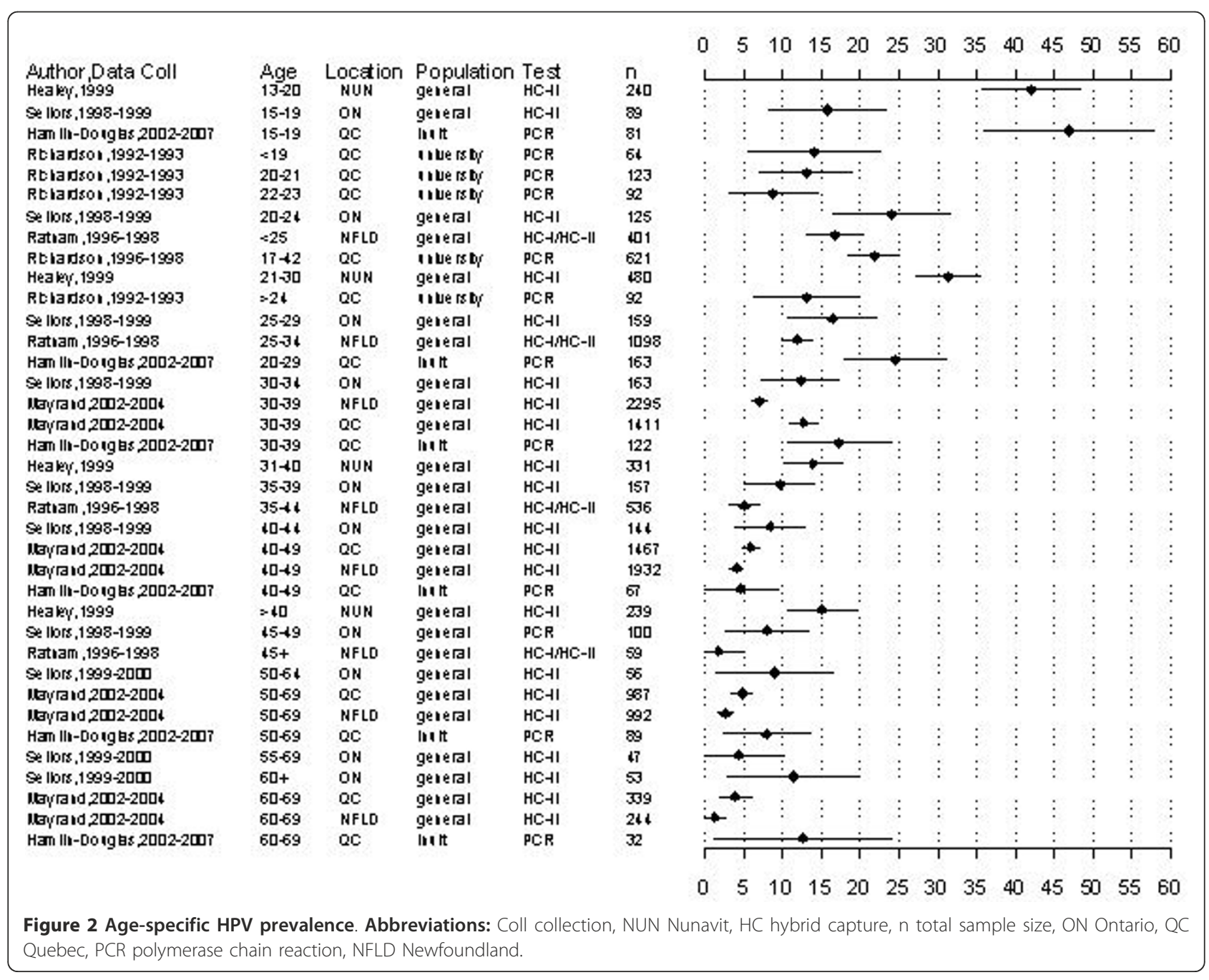




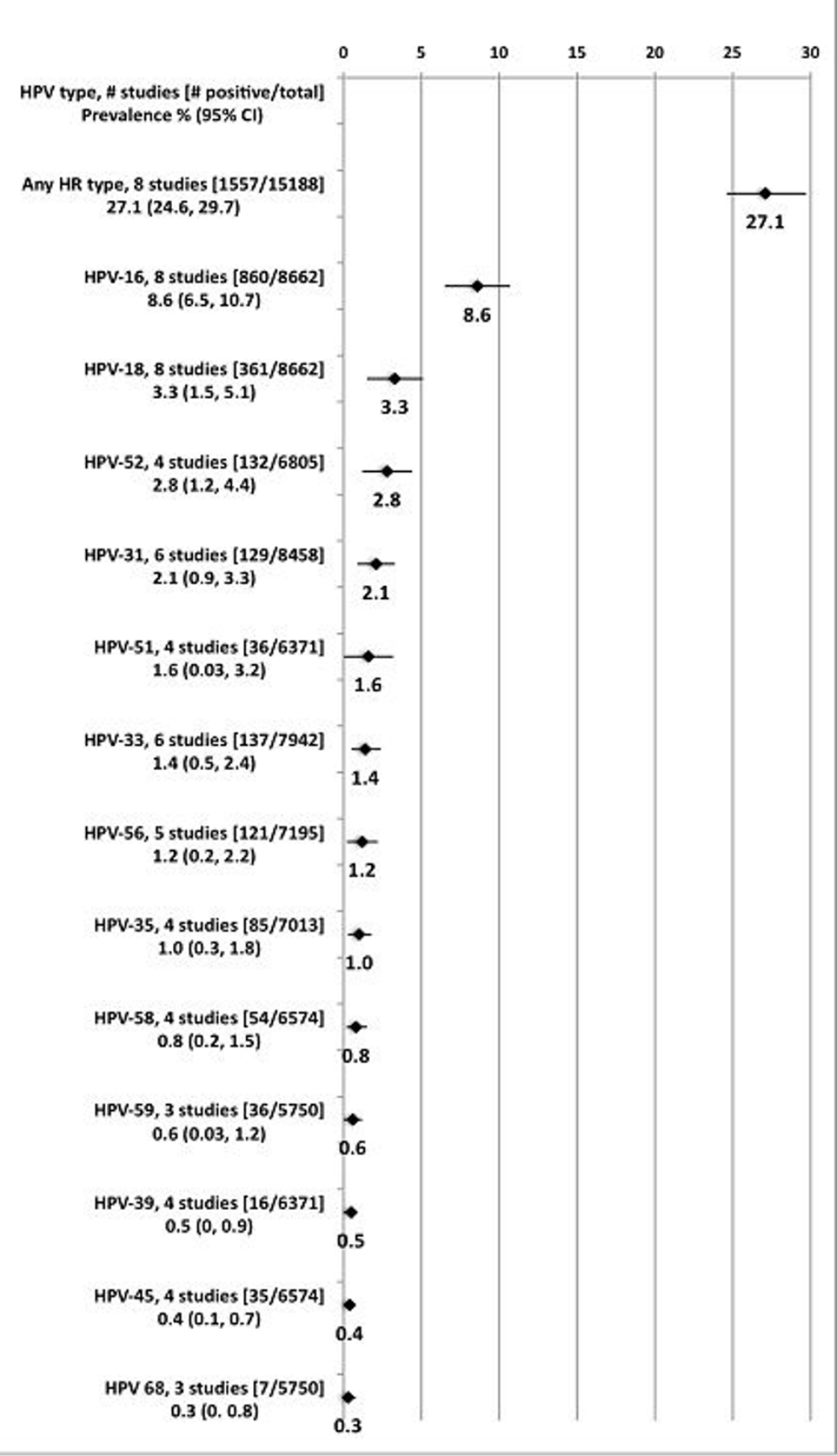

Figure 3 HPV prevalence meta-analyses among all participants. Note: Each point estimate and 95\% confidence interval is a separate metaanalysis. Abbreviations: HR high risk, HPV human papillomavirus, Cl confidence interval.

\section{Discussion}

To our knowledge, this is the most comprehensive systematic review for a single country. Other HPV prevalence reviews included worldwide data and grouped all countries in North America together $[27,28,65]$. The past reviews included few Canadian studies, while data from 30 Canadian studies along with 21 companion reports were included here. Previous reviews focused on HPV prevalence among cervical cancer $[27,28,65]$, HIV [82] or healthy individuals [83]. Our review 


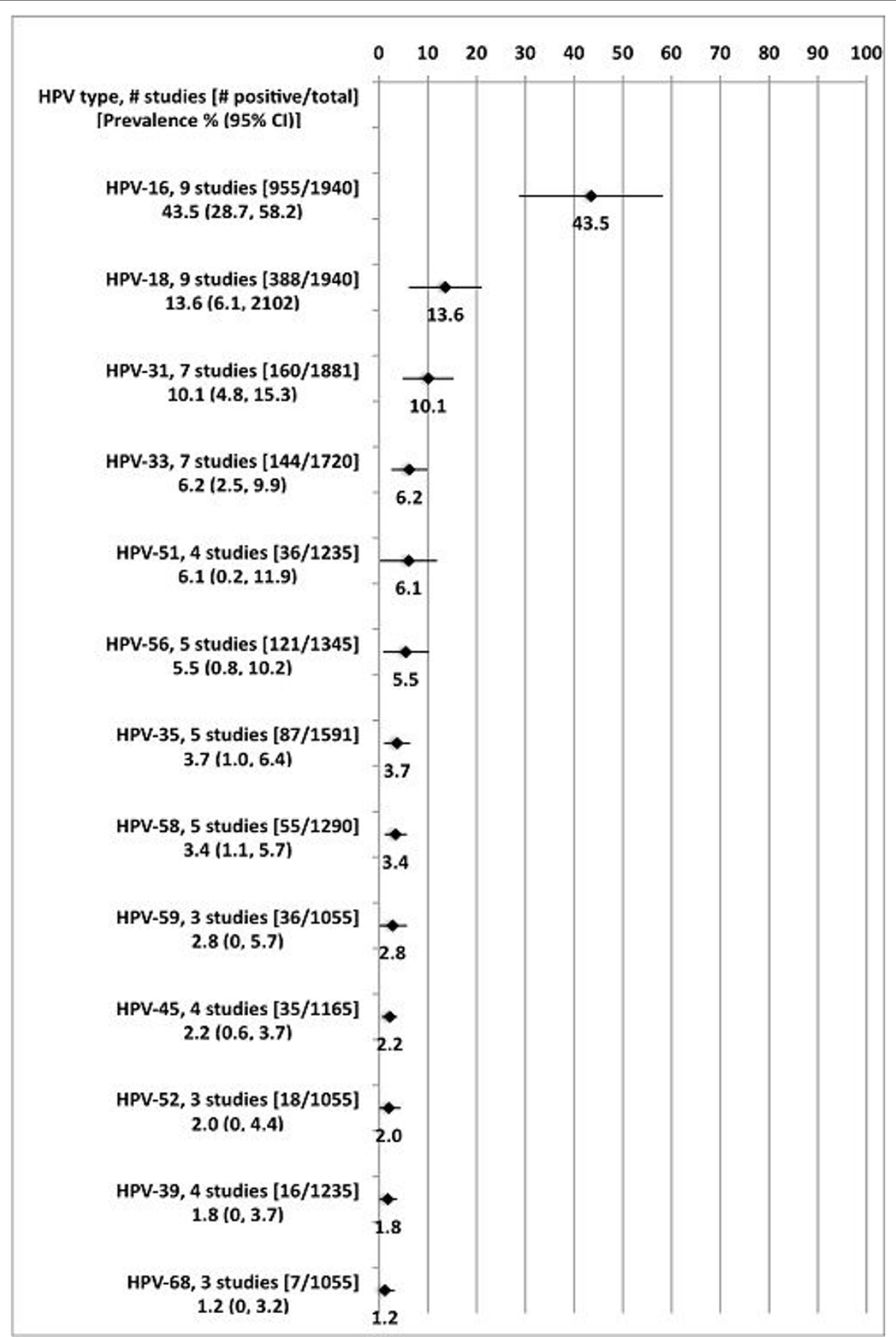

Figure $4 \mathrm{HPV}$ prevalence meta-analyses among HPV-infected participants. Notes: Each point estimate and 95\% confidence interval is a separate meta-analysis. One study (Aho 2003) included in the HPV prevalence among all participants was not included here because only one HPV type was examined (HPV-52). Abbreviations: HPV human papillomavirus, Cl confidence interval.

includes data on the full spectrum of HR HPV infection, providing a more comprehensive understanding of the role of HPV genotypes in different manifestations of infection.
HPV prevalence data are required to provide information related to baseline HPV burden of disease when implementing vaccination programs. All of the included studies were either conducted prior to HPV vaccination 
Table 2 Prevalence of HPV by Bethesda classification system

\begin{tabular}{|c|c|c|c|c|c|c|}
\hline $\begin{array}{l}\text { HPV } \\
\text { Type }\end{array}$ & $\begin{array}{l}\text { Bethesda } \\
\text { classification }\end{array}$ & $\begin{array}{c}\# \\
\text { studies }\end{array}$ & Reference(s) & $\begin{array}{c}\# \\
\text { cases }\end{array}$ & $\begin{array}{l}\text { Total sample } \\
\text { size }\end{array}$ & $\begin{array}{c}\% \text { Prevalence }(95 \% \mathrm{Cl}) \text { from meta- } \\
\text { analysis }\end{array}$ \\
\hline \multirow[t]{6}{*}{$\mathrm{HR} \neq$} & Negativet & 9 & {$[31,35,39,48,49,51,52,54,57]$} & 1451 & 15102 & $40.5 \%(26.4 \%-54.6 \%)$ \\
\hline & Benign & 2 & {$[51]$} & 42 & 275 & $15.7 \%(9.3 \%-22.0 \%)$ \\
\hline & ASC-US & 5 & {$[31,39,48,51,53,54]$} & 136 & 350 & $40.5 \%(26.4 \%-54.6 \%)$ \\
\hline & LSIL & 10 & {$[31,35,39,45,48,49,51,52,54,57]$} & 928 & 1563 & $73.6 \%(55.3 \%-91.8 \%)$ \\
\hline & $\mathrm{HSIL}$ & 8 & {$[31,35,39,45,51,54,57,78]$} & 559 & 628 & $89.9 \%(84.8 \%-95.1 \%)$ \\
\hline & Cervical Cancer & 2 & {$[51,54]$} & 2 & 3 & * \\
\hline \multirow[t]{6}{*}{16} & Negative & 6 & {$[35,39-41,48,52]$} & 473 & 6017 & $7.9 \%(4.9 \%-11.0 \%)$ \\
\hline & Benign & 1 & [39] & 2 & 250 & * \\
\hline & ASC-US & 1 & [48] & 0 & 17 & $*$ \\
\hline & LSIL & 7 & {$[35,39-41,45,48,52]$} & 506 & 1925 & $28.3 \%(15.1 \%-41.5 \%)$ \\
\hline & $\mathrm{HSIL}$ & 7 & {$[35,39-41,45,48,61]$} & 516 & 1068 & $54.0 \%(45.6 \%-62.4 \%)$ \\
\hline & Cervical Cancer & 3 & {$[38,43,61]$} & 81 & 172 & $48.8 \%(34.0 \%-63.6 \%)$ \\
\hline \multirow[t]{6}{*}{18} & Negative & 5 & {$[35,39,40,42,48]$} & 156 & 4891 & $3.6 \%(1.6 \%-5.6 \%)$ \\
\hline & Benign & 1 & [39] & 14 & 250 & $*$ \\
\hline & ASC-US & 1 & [48] & 2 & 17 & * \\
\hline & LSIL & 6 & {$[35,39,40,42,48,52]$} & 81 & 1312 & $5.8 \%(3.6 \%-7.9 \%)$ \\
\hline & HSIL & 5 & {$[35,39,40,42,48]$} & 47 & 662 & $7.9 \%(3.8 \%-12.0 \%)$ \\
\hline & Cervical Cancer & 4 & {$[38,42,43,80]$} & 42 & 219 & $17.1 \%$ (6.4\%-27.9\%) \\
\hline \multirow[t]{6}{*}{31} & Negative & 4 & {$[35,39,40,48]$} & 33 & 4849 & $2.1 \%(0.1 \%-4.1 \%)$ \\
\hline & Benign & 1 & {$[39]$} & 2 & 250 & * \\
\hline & ASC-US & 1 & [48] & 0 & 17 & $*$ \\
\hline & LSIL & 5 & {$[35,39,40,48,52]$} & 76 & 1241 & $7.3 \%(2.7 \%-11.8 \%)$ \\
\hline & $\mathrm{HSIL}$ & 4 & {$[35,39,40,48]$} & 64 & 432 & $14.2 \%(4.0 \%-24.5 \%)$ \\
\hline & Cervical Cancer & 3 & {$[38,43,61]$} & 3 & 172 & $1.2 \%(0.0 \%-2.73 \%)$ \\
\hline \multirow[t]{6}{*}{33} & Negative & 4 & {$[35,39,40,48]$} & 38 & 4849 & $0.6 \%(0.0 \%-1.3 \%)$ \\
\hline & Benign & 1 & [39] & 1 & 250 & * \\
\hline & ASC-US & 1 & [48] & 2 & 17 & $*$ \\
\hline & LSIL & 4 & {$[35,39,40,48]$} & 35 & 884 & $3.6 \%(1.2 \%-6.0 \%)$ \\
\hline & HSIL & 4 & {$[35,39,40,48]$} & 24 & 432 & $5.6 \%(3.5 \%-7.8 \%)$ \\
\hline & Cervical Cancer & 3 & {$[38,43,61]$} & 4 & 172 & $2.1 \%(0.0 \%-4.2 \%)$ \\
\hline \multirow[t]{6}{*}{35} & Negative & 4 & {$[35,39,40,48]$} & 28 & 4849 & $0.5 \%(0.3 \%-0.8 \%)$ \\
\hline & Benign & 1 & [39] & 1 & 250 & * \\
\hline & ASC-US & 1 & {$[48]$} & 0 & 17 & $*$ \\
\hline & LSIL & 4 & {$[35,39,40,48]$} & 31 & 884 & $3.1 \%(0.3 \%-6.0 \%)$ \\
\hline & $\mathrm{HSIL}$ & 4 & {$[35,39,40,48]$} & 8 & 432 & $1.8 \%(0.5 \%-3.0 \%)$ \\
\hline & Cervical Cancer & 3 & {$[38,43,61]$} & 0 & 172 & $*$ \\
\hline \multirow[t]{6}{*}{39} & Negative & 3 & {$[39,40,48]$} & 11 & 4770 & $0.8 \%(0.0 \%-2.0 \%)$ \\
\hline & Benign & 1 & [39] & 0 & 250 & * \\
\hline & ASC-US & 1 & [48] & 1 & 17 & $*$ \\
\hline & LSIL & 3 & {$[39,40,48]$} & 6 & 860 & $0.6 \%(0.0 \%-1.5 \%)$ \\
\hline & HSIL & 3 & {$[39,40,48]$} & 2 & 374 & $*$ \\
\hline & Cervical Cancer & 2 & {$[38,61]$} & 1 & 96 & $*$ \\
\hline \multirow[t]{4}{*}{45} & Negative & 3 & {$[39,40,48]$} & 31 & 4770 & $0.6 \%(0.4 \%-0.9 \%)$ \\
\hline & Benign & 1 & [39] & 0 & 250 & * \\
\hline & ASC-US & 1 & [48] & 0 & 17 & * \\
\hline & LSIL & 3 & {$[39,40,48]$} & 9 & 860 & $1.1 \%(0.4 \%-1.7 \%)$ \\
\hline
\end{tabular}


Table 2 Prevalence of HPV by Bethesda classification system (Continued)

\begin{tabular}{|c|c|c|c|c|c|c|}
\hline & $\mathrm{HSIL}$ & 3 & {$[39,40,48]$} & 6 & 374 & * \\
\hline & Cervical Cancer & 2 & {$[38,61]$} & 8 & 96 & $7.7 \%(2.4 \%-13.0 \%)$ \\
\hline \multirow[t]{6}{*}{51} & Negative & 3 & [39] & 7 & 4470 & * \\
\hline & Benign & 1 & [39] & 0 & 250 & * \\
\hline & ASC-US & 1 & [48] & 1 & 17 & * \\
\hline & LSIL & 3 & {$[39,40,48]$} & 2 & 860 & * \\
\hline & $\mathrm{HSIL}$ & 3 & {$[39,40,48]$} & 3 & 374 & * \\
\hline & Cervical Cancer & 2 & {$[38,61]$} & 0 & 96 & ${ }^{*}$ \\
\hline \multirow[t]{6}{*}{52} & Negative & 4 & {$[39,40,48,52]$} & 41 & 5530 & $1.4 \%(0.1 \%-2.7 \%)$ \\
\hline & Benign & 1 & [39] & 0 & 250 & * \\
\hline & ASC-US & 1 & {$[48]$} & 1 & 17 & * \\
\hline & LSIL & 4 & {$[39,40,48,52]$} & 50 & 1217 & $2.9 \%(0.8 \%-5.1 \%)$ \\
\hline & $\mathrm{HSIL}$ & 3 & {$[39,40,48]$} & 9 & 374 & $2.4 \%(0.9 \%-4.0 \%)$ \\
\hline & Cervical Cancer & 2 & {$[38,61]$} & 3 & 96 & $2.6 \%(0.0 \%-7.1 \%)$ \\
\hline \multirow[t]{6}{*}{56} & Negative & 3 & {$[39,40,48]$} & 74 & 4770 & $1.4 \%(0.8 \%-2.0 \%)$ \\
\hline & Benign & 1 & [39] & 5 & 250 & * \\
\hline & ASC-US & 1 & [48] & 0 & 17 & * \\
\hline & LSIL & 3 & {$[39,40,48]$} & 52 & 860 & $5.8 \%(3.2 \%-8.3 \%)$ \\
\hline & $\mathrm{HSIL}$ & 3 & {$[39,40,48]$} & 4 & 374 & * \\
\hline & Cervical Cancer & 2 & {$[38,61]$} & 2 & 96 & $1.6 \%(0.0 \%-4.1 \%)$ \\
\hline \multirow[t]{6}{*}{58} & Negative & 4 & {$[35,39,40,48]$} & 37 & 4849 & $1.0 \%(0.3 \%-1.6 \%)$ \\
\hline & Benign & 1 & [39] & 0 & 250 & * \\
\hline & ASC-US & 1 & [48] & 0 & 17 & ${ }^{*}$ \\
\hline & LSIL & 4 & {$[35,39,40,48]$} & 22 & 884 & $2.3 \%(1.3 \%-3.3 \%)$ \\
\hline & HSIL & 4 & {$[35,39,40,48]$} & 11 & 432 & $2.0 \%(0.0 \%-4.3 \%)$ \\
\hline & Cervical Cancer & 2 & {$[38,61]$} & 0 & 96 & * \\
\hline \multirow[t]{6}{*}{59} & Negative & 3 & {$[39,40,48]$} & 25 & 4770 & $0.5 \%(0.0 \%-1.1 \%)$ \\
\hline & Benign & 1 & [39] & 2 & 250 & * \\
\hline & ASC-US & 1 & {$[48]$} & 1 & 17 & * \\
\hline & LSIL & 3 & {$[39,40,48]$} & 9 & 860 & $0.9 \%(0.3 \%-1.6 \%)$ \\
\hline & $\mathrm{HSIL}$ & 3 & {$[39,40,48]$} & 9 & 374 & $2.1 \%(0.7 \%-3.6 \%)$ \\
\hline & Cervical Cancer & 2 & {$[38,61]$} & 0 & 96 & * \\
\hline \multirow[t]{6}{*}{68} & Negative & 3 & {$[39,40,48]$} & 5 & 4470 & * \\
\hline & Benign & 1 & [39] & 0 & 250 & * \\
\hline & ASC-US & 1 & {$[48]$} & 0 & 17 & * \\
\hline & LSIL & 3 & {$[39,40,48]$} & 1 & 860 & * \\
\hline & $\mathrm{HSIL}$ & 3 & {$[39,40,48]$} & 2 & 374 & * \\
\hline & Cervical Cancer & 2 & {$[38,61]$} & 1 & 96 & * \\
\hline
\end{tabular}

Notes: *meta-analysis not feasible due to small numbers, tnegative for intraepithelial lesion or malignancy, $\neq$ includes analyses of all HR types combined (i.e., multiple-infection). Abbreviations: atypical squamous cells of undetermined significance (ASCUS), low-grade squamous intraepithelial lesions (LSIL), HSIL highgrade squamous intraepithelial lesions (HSIL).

or did not include vaccinated individuals. These data can be used to evaluate current HPV vaccination program in the future, including vaccine impact on $\mathrm{HR}$ genotype prevalence. The data are also useful for health economic evaluations and epidemiological modeling research. HPV is not a reportable disease so baseline prevalence data could only be obtained by synthesizing highly heterogeneous individual studies. A limited number of studies provide population-based estimates of HPV prevalence and only one provincial based study [39] was available for our meta-analyses.

The results can inform optimal vaccination program scheduling. Our findings indicate that HPV prevalence was highest among females aged less than 20 years. Some 
countries (such as Canada) have publicly funded vaccination programs targeting pre-adolescent and adolescent girls and our results support this approach. High age-specific HR HPV prevalence up to the age of 30 years stresses the need of evaluating the impact of catch-up vaccination programs in these older age groups.

Our results indicate that the prevalence of HR HPV varies among different subgroups of the population with indications of higher prevalence in those at greater risk of being under-screened. In our review, socially disadvantaged individuals (i.e., living in low-income, inner-city areas or Aboriginal communities) had the greatest HPV prevalence. Although declines in incidence and mortality due to routine screening have occurred, [84] false-negative Pap test results continue to raise important questions regarding optimal screening policies $[15,16]$. Our review identified a positive HPV test (e.g., 7.9\% HPV-16 and $3.6 \%$ HPV-18) among females with a negative Pap test. Both tests have associated sensitivities and specificities, and while there is continuing research in identifying the best screening policies with these tests, vaccination remains an effective method to prevent infection with the major HR HPV types [85]. In addition to errors due to diagnostic sensitivity and specificity, $12 \%$ of Canadians never go for a Pap test [9]. Individuals less likely to obtain the Pap test include those of lower education, $[10,12,13]$ lower income, $[10,12,13]$ recent immigrants, $[14,86]$ ethnic minorities, [11] living in rural areas, [11] and those who are obese [87]. These groups of individuals merit special attention when implementing and evaluating HPV vaccination programs.

The HPV vaccines protect against oncogenic strains HPV-16 and -18. These two HPV types contributed a combined prevalence of $12 \%$ for HR HPV infection and $66 \%$ of cervical cancer, which potentially can be prevented by the vaccines assuming $100 \%$ efficacy and long-lasting immunity. These estimates are slightly lower than previous reviews that reported this estimate as being approximately $70 \%,[26]$ indicating that our results might be conservative. Emerging data showing cross-protection against several non-vaccine HR HPV types is encouraging. According to the bivalent vaccine product monograph, protection against HPV-31 (according to protocol cohort) and -45 (total vaccinated cohort) was also observed [17]. These two HR HPV types contributed an estimated combined prevalence of $8.9 \%$ (1.2\% for HPV-31 and 7.7\% for HPV-45) for cervical cancer, indicating that additional cancer protection is a possibility. According to the quadrivalent vaccine product monograph, there was some cross-protection against HPV-31, but not against -45 [18]. Ongoing follow-up of clinical trials and national registries can help understand the potential impact of cross-protection on HPV-related cancer reduction.
Our results are similar to previous reviews of HPV prevalence. The overall HR HPV prevalence observed in our systematic review was $27.1 \%$ among screening populations, which is lower than the prevalence for any HPV infection in HIV positive women (36.3\%) reported in a previous review, as expected [82]. In addition, another previous review found that HPV prevalence peaked in younger women ( $\leq 25$ years of age) and decreased with age, which is consistent with our results [83]. Similar to another review, [65] we found that there was an inconsistent relationship between HPV prevalence in HSIL versus cervical cancer cases across different HPV types. For example, we found a higher HPV prevalence in HSIL versus cervical cancer (including squamous cell carcinoma of the cervix and/or aden/adenosquamous carcinoma of the cervix) for HPV 16 (54.0\% HSIL versus $48.8 \%$ cervical cancer), 31 (14.2\% HSIL versus $1.2 \%$ cervical cancer), and 33 (5.6\% HSIL versus $2.1 \%$ cervical cancer), whereas the reverse was true for HPV 18 (7.9\% HSIL versus $17.1 \%$ cervical cancer) and 52 (2.4\% HSIL versus $2.6 \%$ cervical cancer). The relationship between HSIL and cervical cancer could not be explored for the other HPV types, due to insufficient data. Furthermore, many of the cervical cancer estimates were based on a small number of studies, making these results difficult to interpret.

We found that multiple infections occurred in $17.1 \%$ of the included prevalence estimates. Multiple infections occurred in $11.2 \%$ of the prevalence estimates in a review of invasive cervical cancer [26] and $11.9 \%$ in a review limited to women infected with HIV [82]. Factors that can explain the difference in multiple infections across reviews include different duration of specific types of infection (i.e., natural clearance of one or several types but persistence of the main one); and/or potentially different number of new sexual partners during the last months in females with LSIL, HSIL and those with cervical cancer or HIV.

Compared to different regions throughout the world, HPV prevalence in Canada is moderate. One review found that the highest prevalence of HPV among middle-aged women (35-50 years) was observed for Africa, Central and South America, and the United States (approximately $20 \%$ ), while a lower prevalence was observed in Asia, Australia, Europe, Middle East, and Canada (approximately 15\%) [83]. Furthermore, the proportion of cancer cases associated with HPV 16 and 18 was found to be the highest in Africa (94.2\%), moderate in North America (89.2\%), and the lowest in Asia (68\%) [26].

Limitations of this systematic review include that the meta-analyses were based on each HPV type individually so may include concomitant infection. Data were also pooled across studies using different tests to measure the presence of HPV (e.g., hybridization, PCR, HC) and these tests vary in their sensitivity and specificity. For 
example, HC-II has been found to be a more sensitive test versus HC-I [88]. The methodological quality of the included studies was variable; less than half used a representative sampling strategy and many had small sample sizes.

Population-based, high-quality, HPV prevalence studies are warranted. These studies should report age-specific prevalence, overlap between single and multiple infection, and HPV type-specific infection. A recently published prevalence study from the United Kingdom is an excellent example of such a study [89]. These data can lead to a better understanding of HPV prevalence, as well as the impact of new prevention programs at the national and international levels.

\section{Conclusions}

Prevalence data of cervical human papillomavirus infection is necessary to inform optimal prevention programs and evaluate current prevention strategies. Previous HPV prevalence reviews included worldwide studies and focused on HPV prevalence among cervical cancer, HIV or healthy individuals. To our knowledge, this is the most comprehensive systematic review for a single country and the data can be used in health economic evaluations and epidemiological modeling research to help inform public health policy. Our results show that the highest prevalence occurred among females $<20$ years of age, yet prevalence remained high among women up to 30 years of age.

\section{Additional material}

Additional file 1: Search strategy for MEDLINE. Search strategy used

in systematic review.

Additional file 2: Methodological quality and risk of bias tool. Tool used to assess methodological quality of studies included in systematic review.

Additional file 3: Bethesda classification system. Classification system for cervical cancer used in analysis of results.

Additional file 4: Methodological quality of the included studies Results of the methodological quality tool.

\section{Acknowledgements and Funding}

We thank Maraki Fikre Merid for her assistance with drafting the introduction on a previous version of this manuscript, Dr Minh Do for his assistance with the data abstraction and screening on a previous version of this review, Natasha Nanwa for her administrative assistance, Maggie Hong Chen for assisting with the statistical analysis, and Dr Jessie McGowan for the literature searches. Funding for this systematic review was received from GlaxoSmithKline, Canada.

\section{Author details}

${ }^{1}$ Li Ka Shing Knowledge Institute, St Michael's Hospital, (38 Shuter Street), Toronto, Ontario, (M5B 1T8), Canada. ${ }^{2}$ School of Population and Public Health, University of British Columbia, (2206 East Mall), Vancouver, British Columbia, (V6T 1Z3), Canada. ${ }^{3}$ Centre de Recherche du CHUL (CHUQ), I'Université Laval, (2705 boulevard Laurier), Québec, Québec, (G1V 4G2), Canada. ${ }^{4}$ GlaxoSmithKline Biologicals, (Avenue Fleming 20), Wavre (1300),
Belgium. ${ }^{5}$ Health Policy Management and Evaluation, University of Toronto, (155 College Street), Toronto, Ontario, (M5T 3MT), Canada. ${ }^{6}$ Toronto Health Economics and Technology Assessment, University of Toronto, (144 College Street), Toronto, Ontario, (M5S 3M2), Canada. ${ }^{7}$ Department of Epidemiology, University of Western Ontario, (Kresge Building), London, Ontario, (N6A 5C1), Canada.

\section{Authors' contributions}

ACT designed and coordinated the study, participated in data collection and analysis, and drafted the manuscript. CHN participated in data collection and analysis and helped to draft the manuscript. VG helped to draft the manuscript. AA helped to draft the manuscript. BP conceived of the study, participated the study design and coordination, and helped to draft the manuscript. SB participated in data collection and analysis and helped to draft the manuscript. All authors read and approved the final manuscript.

\section{Competing interests}

ACT, CHN, and SC have been paid consultants for vaccine-related products from GlaxoSmithKline, Canada. VG has received consultancy fees from GlaxoSmithKline and Merck Frosst Canada. AA is an employee of GlaxoSmithKline Biologicals. The funding agreement ensured the authors' independence in designing the study, interpreting the data, writing, and publishing the report.

Received: 1 May 2011 Accepted: 5 September 2011 Published: 5 September 2011

\section{References}

1. Schiffman M, Kjaer SK: Chapter 2: Natural history of anogenital human papillomavirus infection and neoplasia. J Natl Cancer Inst Monogr 2003, 14-19.

2. Munoz N, Bosch FX, de SS, Herrero R, Castellsague X, Shah KV, Snijders PJ, Meijer CJ: Epidemiologic classification of human papillomavirus types associated with cervical cancer. $N$ Engl J Med 2003, 348:518-527.

3. Bouvard V, Baan R, Straif K, Grosse Y, Secretan B, El GF, brahim-Tallaa L, Guha N, Freeman C, Galichet L, Cogliano V: A review of human carcinogens-Part B: biological agents. Lancet Oncol 2009, 10:321-322.

4. Walboomers JM, Jacobs MV, Manos MM, Bosch FX, Kummer JA, Shah KV, Snijders PJ, Peto J, Meijer CJ, Munoz N: Human papillomavirus is a necessary cause of invasive cervical cancer worldwide. J Pathol 1999, 189:12-19.

5. Bernard HU, Calleja-Macias IE, Dunn ST: Genome variation of human papillomavirus types: phylogenetic and medical implications. Int J Cancer 2006, 118:1071-1076.

6. Aubin F, Pretet $J$, Jacquard $A C$, Saunier $M$, Carcopino $X$, Jaroud $F$, Pradat $P$, Soubeyrand B, Leocmach Y, Mougin C, Riethmuller D: Human papillomavirus genotype distribution in external acuminata condylomata: a Large French National Study (EDiTH IV). Clin Infect Dis 2008, 47:610-615.

7. Shepherd $\sqcup$, Bryson SC: Human papillomavirus-lessons from history and challenges for the future. J Obstet Gynaecol Can 2008, 30:1025-1033.

8. Canadian Cancer Society's Steering Committee: Canadian cancer statistics 2009. Toronto 2009.

9. Health Canada: Cervical cancer screening in Canada: 1998 Surveillance report. Ottawa 2002.

10. Gupta S, Roos LL, Walld R, Traverse D, Dahl M: Delivering equitable care: comparing preventive services in Manitoba. Am J Public Health 2003, 93:2086-2092.

11. Johnston GM, Boyd CJ, Maclsaac MA: Community-based cultural predictors of Pap smear screening in Nova Scotia. Can J Public Health 2004, 95:95-98.

12. Katz SJ, Hofer TP: Socioeconomic disparities in preventive care persist despite universal coverage. Breast and cervical cancer screening in Ontario and the United States. JAMA 1994, 272:530-534.

13. Lofters A, Glazier RH, Agha MM, Creatore MI, Moineddin R: Inadequacy of cervical cancer screening among urban recent immigrants: a population-based study of physician and laboratory claims in Toronto, Canada. Prev Med 2007, 44:536-542.

14. McDonald JT, Kennedy S: Cervical cancer screening by immigrant and minority women in Canada. J Immigr Minor Health 2007, 9:323-334. 
15. Cuzick J, Clavel C, Petry KU, Meijer CJ, Hoyer H, Ratnam S, Szarewski A, Birembaut $P$, Kulasingam $S$, Sasieni $P$, Iftner T: Overview of the European and North American studies on HPV testing in primary cervical cancer screening. Int J Cancer 2006, 119:1095-1101.

16. Nanda K, McCrory DC, Myers ER, Bastian LA, Hasselblad V, Hickey JD, Matchar DB: Accuracy of the Papanicolaou test in screening for and follow-up of cervical cytologic abnormalities: a systematic review. Ann Intern Med 2000, 132:810-819.

17. Cervarix ${ }^{\mathrm{TM}}$ Product Monograph, August 2010.

18. Gardasil|TM Product Monograph, May 2011.

19. Franco EL, Ferenczy A: Cervical cancer screening following the implementation of prophylactic human papillomavirus vaccination. Future Oncol 2007, 3:319-327.

20. Franco EL, Cuzick J, Hildesheim A, de SS: Chapter 20: Issues in planning cervical cancer screening in the era of HPV vaccination. Vaccine 2006, 24(Suppl 3):S3171-S3/177.

21. Erickson LJ, De WP, Farand L: An analytical framework for immunization programs in Canada. Vaccine 2005, 23:2470-2476.

22. Howlett RI, Miller AB, Pasut G, Mai V: Defining a strategy to evaluate cervical cancer prevention and early detection in the era of HPV vaccination. Prev Med 2009, 48:432-437.

23. Higgins JPT, Green S, editors: Cochrane Handbook for Systematic Reviews of Interventions The Cochrane Collaboration; 2009 [http://www.cochranehandbook.org], Version 5.0.2 [updated September 2009].

24. Moher D, Liberati A, Tetzlaff J, Altman DG: Preferred reporting items for systematic reviews and meta-analyses: the PRISMA statement. BMJ 2009, 339:b2535.

25. Sampson M, McGowan J, Lefebvre C, Moher D, Grimshaw J: PRESS: Peer Review of Electronic Search Strategies. Ottawa 2008.

26. Li N, Franceschi S, Howell-Jones R, Snijders PJ, Clifford GM: Human papillomavirus type distribution in 30,848 invasive cervical cancers worldwide: Variation by geographical region, histological type and year of publication. Int J Cancer 2010.

27. Clifford GM, Smith JS, Plummer M, Munoz N, Franceschi S: Human papillomavirus types in invasive cervical cancer worldwide: a metaanalysis. Br J Cancer 2003, 88:63-73.

28. Smith JS, Lindsay L, Hoots B, Keys J, Franceschi S, Winer R, Clifford GM: Human papillomavirus type distribution in invasive cervical cancer and high-grade cervical lesions: a meta-analysis update. Int J Cancer 2007, 121:621-632.

29. Duggan MA, Inoue M, McGregor SE, Nation JG: A comparison of cervical scrapes for HPV typing by dot-blot hybridization obtained by wood and plastic spatulas. Journal of Virological Methods 1990, 29:267-277.

30. Peng HQ, Roth P, Caussy D, Rawls W: Comparison of the Cytobrush and cotton swabs in sampling cervical cells for filter in situ hybridization detection of human papillomavirus types 16 and 18 DNA. Acta cytologica 1988, 32:311-313.

31. Sellors JW, Mahony JB, Kaczorowski J, Lytwyn A, Bangura H, Chong S, Lorincz A, Dalby DM, Janjusevic V, Keller JL: Prevalence and predictors of human papillomavirus infection in women in Ontario, Canada. Survey of HPV in Ontario Women (SHOW) Group.[see comment]. CMAJ Canadian Medical Association Journal 2000, 163:503-508

32. Sellors JW, Karwalajtys TL, Kaczorowski JA, Mahony JB, Lytwyn A, Chong S, Sparrow J, Lorincz A, Survey of HPV in Older Ontario Women (SHOOW) Group: Prevalence of infection with carcinogenic human papillomavirus among older women. CMAJ Canadian Medical Association Journal 2002, 167:871-873.

33. Sanderson S, Tatt ID, Higgins JP: Tools for assessing quality and susceptibility to bias in observational studies in epidemiology: a systematic review and annotated bibliography. Int J Epidemiol 2007, 36:666-676.

34. Bishop FL, Prescott P, Chan YK, Saville J, von EE, Lewith GT: Prevalence of complementary medicine use in pediatric cancer: a systematic review. Pediatrics 2010, 125:768-776.

35. Sellors JW, Lorincz AT, Mahony JB, Mielzynska I, Lytwyn A, Roth P, Howard M, Chong S, Daya D, Chapman W, Chernesky M: Comparison of self-collected vaginal, vulvar and urine samples with physician-collected cervical samples for human papillomavirus testing to detect high-grade squamous intraepithelial lesions. CMAJ Canadian Medical Association Journal 2000, 163:513-518.

36. Solomon D, Davey D, Kurman R, Moriarty A, O'Connor D, Prey M, Raab S, Sherman M, Wilbur D, Wright T Jr, Young N: The 2001 Bethesda System: terminology for reporting results of cervical cytology. JAMA 2002, 287:2114-2119

37. DerSimonian R, Laird N: Meta-analysis in clinical trials. Control Clin Trials 1986, 7:177-188.

38. Bosch FX, Manos MM, Munoz N, Sherman M, Jansen AM, Peto J, Schiffman MH, Moreno V, Kurman R, Shah KV: Prevalence of human papillomavirus in cervical cancer: a worldwide perspective. International biological study on cervical cancer (IBSCC) Study Group. J Natl Cancer Inst 1995, 87:796-802.

39. Moore RA, Ogilvie G, Fornika D, Moravan V, Brisson M, mirabbasi-Beik M, Kollar A, Burgess T, Hsu R, Towers L, Lo J, Matisic J, Brooks-Wilson A: Prevalence and type distribution of human papillomavirus in 5,000 British Columbia women-implications for vaccination. Cancer Causes Control 2009, 20:1387-1396.

40. Antonishyn NA, Horsman GB, Kelln RA, Saggar J, Severini A: The impact of the distribution of human papillomavirus types and associated high-risk lesions in a colposcopy population for monitoring vaccine efficacy. Arch Pathol Lab Med 2008, 132:54-60.

41. Brisson J, Morin C, Fortier M, Roy M, Bouchard C, Leclerc J, Christen A, Guimont C, Penault F, Meisels A: Risk factors for cervical intraepithelial neoplasia: differences between low- and high-grade lesions. American Journal of Epidemiology 1994, 140:700-710.

42. Caussy D, Marrett LD, Worth AJ, McBride M, Rawls WE: Human papillomavirus and cervical intraepithelial neoplasia in women who subsequently had invasive cancer. CMAJ Canadian Medical Association Journal 1990, 142:311-317.

43. Duggan MA, McGregor SE, Benoit JL, Inoue M, Nation JG, Stuart GC: The human papillomavirus status of invasive cervical adenocarcinoma: a clinicopathological and outcome analysis. Hum Pathol 1995, 26:319-325.

44. Duggan MA, McGregor SE, Stuart GC, Morris S, Chang-Poon V, Schepansky A, Honore L: The HPV determinants of CIN I. European Journal of Gynaecological Oncology 1997, 18:117-123.

45. Feoli-Fonseca JC, Oligny LL, Brochu P, Simard P, Falconi S, Yotov WV: Human papillomavirus (HPV) study of 691 pathological specimens from Quebec by PCR-direct sequencing approach. Journal of Medical Virology 2001, 63:284-292.

46. Franco E, Bergeron J, Villa L, Arella M, Richardson L, Arseneau J, Stanimir G: Human papillomavirus DNA in invasive cervical carcinomas and its association with patient survival: a nested case-control study. Cancer Epidemiology, Biomarkers \& Prevention 1996, 5:271-275.

47. Guijon F, Paraskevas M, McNicol P: Human papillomavirus infection and the size and grade of cervical intraepithelial neoplastic lesions associated with failure of therapy. International Journal of Gynaecology \& Obstetrics 1993, 42:137-142.

48. Hamlin-Douglas L: Prevalence and determinants of human papillomavirus (HPV) infection in Inuit women of Nunavik, Quebec. PhD Thesis McGill University; 2008.

49. Hankins C, Coutlee F, Lapointe N, Simard P, Tran T, Samson J, Hum L: Prevalence of risk factors associated with human papillomavirus infection in women living with HIV. Canadian Women's HIV Study Group. CMAJ Canadian Medical Association Journal 1999, 160:185-191.

50. Healey SM, Aronson KJ, Mao Y, Schlecht NF, Mery LS, Ferenczy A, Franco EL: Oncogenic human papillomavirus infection and cervical lesions in aboriginal women of Nunavut, Canada. Sexually Transmitted Diseases 2001, 28:694-700.

51. Kapala J, Jang D, Patel J, Biers K, Smieja M, Chernesky M: Pap cytopathology and the presence of high-risk human papillomavirus in SurePath liquid preservative and Digene cervical sampler specimens. Journal of Virological Methods 2007, 142:223-225.

52. Koushik A, Ghosh A, Duarte-Franco E, Forest P, Voyer H, Matlashewski G, Coutlee F, Franco EL, Biomarkers of Cervical Cancer Risk (BCCR) Study Team: The p53 codon 72 polymorphism and risk of high-grade cervical intraepithelial neoplasia. Cancer Detection \& Prevention 2005, 29:307-316.

53. Lytwyn A, Sellors JW, Mahony JB, Daya D, Chapman W, Ellis N, Roth P, Lorincz AT, Gafni A: Comparison of human papillomavirus DNA testing and repeat Papanicolaou test in women with low-grade cervical cytologic abnormalities: a randomized trial. HPV Effectiveness in Lowgrade Paps (HELP) Study No. 1 Group. CMAJ Canadian Medical Association Journal 2000, 163:701-707.

54. Mayrand MH, Duarte FE, Coutlée F, Rodrigues I, Walter SD, Ratnam S, Franco EL, CCCaST-Study-Group: Randomized controlled trial of human 
papillomavirus testing versus Pap cytology in the primary screening for cervical cancer precursors: design, methods and preliminary accrual results of the Canadian cervical cancer screening trial (CCCaST). International journal of cancer Journal international du cancer 2006, 119:615-623.

55. Michael P: PCR screening for human papillomavirus infections, and evaluation of the estimated infection prevalence for a population of females. PhD Thesis Laurentian University; 1999.

56. Ogilvie G, Krajden M, Maginley J, Isaac-Renton J, Hislop G, Elwood-Martin R, Sherlock C, Taylor D, Rekart M: Feasibility of self-collection of specimens for human papillomavirus testing in hard-to-reach women. CMAJ Canadian Medical Association Journal 2007, 177:480-483.

57. Ratnam S, Hogan K, Hankins C: Prevalence of HIV infection among pregnant women in Newfoundland. CMAJ: Canadian Medical Association Journal 1996, 154:1027-1032.

58. Richardson H, Franco E, Pintos J, Bergeron J, Arella M, Tellier P: Determinants of low-risk and high-risk cervical human papillomavirus infections in Montreal University students. Sexually Transmitted Diseases 2000, 27:79-86.

59. Richardson $H$, Kelsall $G$, Tellier P, Voyer $H$, Abrahamowicz M, Ferenczy A, Coutlee F, Franco EL: The natural history of type-specific human papillomavirus infections in female university students. Cancer Epidemiology, Biomarkers \& Prevention 2003, 12:485-490

60. Rohan T, Mann V, McLaughlin J, Harnish DG, Yu H, Smith D, Davis R, Shier RM, Rawls W: PCR-detected genital papillomavirus infection: prevalence and association with risk factors for cervical cancer. Int J Cancer 1991, 49:856-860.

61. Tran-Thanh D, Koushik A, Provencher D, Drouin P, Dubuc-Lissoir J, Gauthier P, Allaire G, Kornegay J, Franco E, Coutlee F: Detection of human herpes virus type 6 DNA in precancerous lesions of the uterine cervix. J Med Virol 2002, 68:606-610.

62. Young TK, McNicol P, Beauvais J: Factors associated with human papillomavirus infection detected by polymerase chain reaction among urban Canadian aboriginal and non-aboriginal women.[see comment]. Sexually Transmitted Diseases 1997, 24:293-298.

63. Aho J, Hankins C, Tremblay C, Lang F, Forest P, Pourreaux K, Rouah F, Coutlee F: Molecular analysis of human papillomavirus type 52 isolates detected in the genital tract of human immunodeficiency virusseropositive and -seronegative women. J Infect Dis 2003, 188:1517-1527.

64. Aho J, Hankins C, Tremblay C, Forest P, Pourreaux K, Rouah F, Coutlee F: Genomic polymorphism of human papillomavirus type 52 predisposes toward persistent infection in sexually active women. J Infect Dis 2004, 190:46-52.

65. Clifford GM, Smith JS, Aguado T, Franceschi S: Comparison of HPV type distribution in high-grade cervical lesions and cervical cancer: a metaanalysis. Br J Cancer 2003, 89:101-105.

66. Coutlee $\mathrm{F}$, Hankins $\mathrm{C}$, Lapointe $\mathrm{N}$ : Comparison between vaginal tampon and cervicovaginal lavage specimen collection for detection of human papillomavirus DNA by the polymerase chain reaction. The Canadian Women's HIV Study Group. Journal of Medical Virology 1997, 51:42-47.

67. Duggan MA, Inoue M, McGregor SE, Stuart GC, Morris S, Chang-Poon V, Schepansky A, Honore L: A paired comparison of dot blot hybridization and PCR amplification for HPV testing of cervical scrapes interpreted as CIN 1. European Journal of Gynaecological Oncology 1994, 15:178-187.

68. Duggan MA, Benoit JL, MCGregor SE, Nation JG, Inoue M, Stuart GC: The human papillomavirus status of 114 endocervical adenocarcinoma cases by dot blot hybridization. Hum Pathol 1993, 24:121-125.

69. Duggan MA, McGregor SE, Stuart GC, Morris S, Chang-Poon V, Schepansky A, Honore L: The natural history of CIN I lesions. European Journal of Gynaecological Oncology 1998, 19:338-344.

70. Duggan MA, McGregor SE, Stuart GC, Morris S, Chang-Poon V, Schepansky A, Honore L: Predictors of co-incidental CIN II/III amongst a cohort of women with CIN I detected by a screening Pap test. European Journal of Gynaecological Oncology 1998, 19:209-214.

71. Gagnon S, Hankins C, Tremblay C, Pourreaux K, Forest P, Rouah F, Coutlee F, The Canadian Women's HIV Study Group: Polymorphism of human papillomavirus type 31 isolates infecting the genital tract of HIVseropositive and HIV-seronegative women at risk for HIV infection. Journal of Medical Virology 2005, 75:213-221.

72. Healey SM: Human papillomavirus infection and cervical dysplasia in Nunavut. PhD Thesis Queen's University; 2000.
73. Healey SM, Aronson K, Mao Y, Franco EL: Human papillomavirus and cervical dysplasia in Nunavut: prelude to a screening strategy. International Journal of Circumpolar Health 2004, 63(Suppl):201.

74. Karwalajtys T, Howard M, Sellors JW, Kaczorowski J: Vaginal self sampling versus physician cervical sampling for HPV among younger and older women. Sexually Transmitted Infections 2006, 82:337-339.

75. Lytwyn A, Sellors JW, Mahony JB, Daya D, Chapman W, Howard M, Roth P Lorincz AT, Gafni A, Walter SD: Adjunctive human papillomavirus testing in the 2-year follow-up of women with low-grade cervical cytologic abnormalities: a randomized trial and economic evaluation. Archives of pathology \& laboratory medicine 2003, 127:1169-1175.

76. Mahmud SM, Koushik A, Duarte-Franco E, Costa J, Fontes G, Bicho M, Coutlee F, Franco EL, Biomarkers of Cervical Cancer Risk Study Team: Haptoglobin phenotype and risk of cervical neoplasia: a case-control study. Clinica Chimica Acta 2007, 385:67-72.

77. Oligny LL, Feoli-Fonseca JC, Brochu P, Simard P, Falconi S, Yotov W: Human papillomavirus PCR direct sequencing study of cervical precancerous lesions in Quebec children. Sexually Transmitted Infections 2001, 77:391.

78. Schaffer A, Koushik A, Trottier H, Duarte-Franco E, Mansour N, Arseneau J, Provencher D, Gilbert L, Gotlieb W, Ferenczy A, Coutlee F, Pollak MN, Franco EL: Insulin-like growth factor-I and risk of high-grade cervical intraepithelial neoplasia. Cancer Epidemiol Biomarkers Prev 2007, 16:716-722.

79. Sellors JW, Karwalajtys TL, Kaczorowski J, Mahony JB, Lytwyn A, Chong S, Sparrow J, Lorincz A, Survey of HPV in Ontario Women Group: Incidence, clearance and predictors of human papillomavirus infection in women. [see comment][comment]. CMAJ Canadian Medical Association Journal 2003, 168:421-425

80. Tran-Thanh D, Provencher D, Koushik A, Duarte-Franco E, Kessous A, Drouin P, Wheeler CM, Dubuc-Lissoir J, Gauthier P, Allaire G, Vauclair R, Dipaolo JA, Gravitt P, Franco E, Coutlee F: Herpes simplex virus type II is not a cofactor to human papillomavirus in cancer of the uterine cervix. Am J Obstet Gynecol 2003, 188:129-134.

81. Richardson H, Abrahamowicz M, Tellier PP, Kelsall G, du BR, Ferenczy A, Coutlee F, Franco EL: Modifiable risk factors associated with clearance of type-specific cervical human papillomavirus infections in a cohort of university students. Cancer Epidemiology, Biomarkers \& Prevention 2005, 14:1149-1156.

82. Clifford GM, Goncalves MA, Franceschi S: Human papillomavirus types among women infected with HIV: a meta-analysis. AIDS 2006, 20:2337-2344.

83. Smith JS, Melendy A, Rana RK, Pimenta JM: Age-specific prevalence of infection with human papillomavirus in females: a global review. J Adolesc Health 2008, 43:S5-25, S25.

84. Marrett LD, Innes M, Howlett R, Cotterchio M: Insight on Cancer: News and information on cervical cancer. Toronto 2005.

85. National Advisory Committee on Immunization (NACI): National Advisory Committee on Immunization (NACl) statement on human papillomavirus vaccine 2007.

86. Woltman KJ, Newbold KB: Immigrant women and cervical cancer screening uptake: a multilevel analysis. Can J Public Health 2007, 98:470-475.

87. Maruthur NM, Bolen SD, Brancati FL, Clark JM: The association of obesity and cervical cancer screening: a systematic review and meta-analysis. Obesity (Silver Spring) 2009, 17:375-381.

88. Kuhn L, Denny L, Pollack A, Lorincz A, Richart RM, Wright TC: Human papillomavirus DNA testing for cervical cancer screening in low-resource settings. J Natl Cancer Inst 2000, 92:818-825.

89. Howell-Jones R, Bailey A, Beddows S, Sargent A, de SN, Wilson G, Anton J, Nichols T, Soldan K, Kitchener H: Multi-site study of HPV type-specific prevalence in women with cervical cancer, intraepithelial neoplasia and normal cytology, in England. Br J Cancer 2010, 103:209-216.

\section{Pre-publication history}

The pre-publication history for this paper can be accessed here: http://www.biomedcentral.com/1471-2334/11/235/prepub

doi:10.1186/1471-2334-11-235

Cite this article as: Tricco et al:: Canadian oncogenic human papillomavirus cervical infection prevalence: Systematic review and meta-analysis. BMC Infectious Diseases 2011 11:235. 